\title{
Depinning of a Superfluid Vortex Inside a Circular Defect
}

\author{
José A. Freire \\ Departamento de Física, Universidade Federal do Paraná, \\ Caixa Postal 19081, Curitiba-PR 81531-990, BRAZIL
}

(October 31, 2018)

\begin{abstract}
In this work we study the process of depinning of a quantum of circulation trapped inside a disk by an applied two dimensional superflow. We use the Gross-Pitaevskii model to describe the neutral superfluid. The collective coordinate dynamics is derived directly from the condensate equation of motion, the nonlinear Schrödinger equation, and it is used to obtain an expression for the critical velocity as a function of the defect radius. This expression is compared with a numerical result obtained from the time independent nonlinear Schrödinger equation. Below the critical velocity, we obtain the dependence of the semiclassical nucleation rate with the flow velocity at infinity. Above the critical velocity, the classical vortex depinning is illustrated with a numerical simulation of the time dependent nonlinear Schrödinger equation.

67.57.De,67.40.Hf,47.37.+q
\end{abstract}

\section{INTRODUCTION}

A superflow passing through a constriction or an obstacle of some sort can produce vorticity in a process known as inhomogeneous nucleation [1,2]. The vortex is created at the boundary between the superfluid and the container walls and two flow regimes are identified. When the flow velocity, far away from the walls, is smaller than a critical velocity an effective barrier is present that prevents vorticity to appear in the flow, this is the laminar flow regime. Above the critical velocity, a vortexless state can dynamically produce vorticity. In the laminar flow regime, and in the absence of thermal excitations, it is believed that vorticity can still be produced via Macroscopic Quantum Tunneling (MQT).

Measurements of the vortex production rates for a sphere moving in a superfluid [3] and for an oscillating superflow through a microscopic orifice [4,5] have shown evidence of MQT of vorticity at sufficiently low temperatures. Numerical studies of the dynamical nucleation process, above the critical velocity, have relied on the nonlinear Schrödinger model for the superfluid dynamics [6,7]. Analytical studies of the tunneling process have used the collective coordinate approach to map what is really a field theory tunneling problem into a standard Quantum Mechanical problem for the vortex coordinates [8]. Besides the bosonic superfluid, similar ideas have been applied to vortex states in short coherence length superconductors [9 12].

Different from Ref. [6], that studied the classical nucleation of a vortex/antivortex pair in the proximity of a circular obstacle in a two dimensional superflow, in this work we will investigate the depinning of a quantum of circulation trapped inside the obstacle.

In the following sections we define the Gross-Pitaevskii model and extract the dynamics of the collective coordinate from the condensate equations of motion. We obtain an expression for the critical velocity and compare it with a numerical result. The process of dynamical nucleation, above the critical velocity, is illustrated with a numerical integration of the nonlinear Schrödinger equation. The Macroscopic Quantum Tunneling, below the critical velocity, is studied within the collective coordinate approach and the semiclassical nucleation rate is obtained.

\section{THE MODEL}

The problem we consider is that of a two dimensional superflow past an impenetrable disk perpendicular to the $z$ axis. We will be interested in the process of removal of a preexistent quantum of vorticity inside the disk by the applied superflow (along the $y$ direction). In three dimensions this would correspond to a straight vortex depinning from a columnar defect due to an applied superflow perpendicular to the column axis. In Ref. [12] this problem is studied considering that the vortex depins in the form of an arc attached to the column.

We start from the Gross-Pitaevskii model for the dynamics of the condensate wave function, i.e. the nonlinear Schrödinger equation (NLSE) 13,14:

$$
i \hbar \frac{\partial \psi}{\partial t}=-\frac{\hbar^{2}}{2 m} \nabla^{2} \psi+g\left(|\psi|^{2}-\rho_{0}\right) \psi .
$$


Here $m$ is the boson mass, $g$ is the parameter in the hard core potential between the bosons and $\rho_{0}$ is the equilibrium superfluid number density. The obstacle and any applied flow impose appropriate boundary conditions on the condensate wave function $\psi$.

It will prove convenient to express length in units of the coherence length $\xi=\sqrt{\hbar^{2} / g \rho_{0} m}$, the condensate in units of $\sqrt{\rho_{0}}$ and velocities in units of the speed of sound $c=\hbar / m \xi$.

We take as boundary conditions on $\psi$ :

$$
\begin{gathered}
\psi(\mathbf{r}, t) \rightarrow e^{i v_{\infty} y} e^{i \theta}, \quad r \rightarrow \infty, \\
\psi(\mathbf{r}, t)=0, \quad r \leq a .
\end{gathered}
$$

The first and second exponentials in 2 say that at infinity we have the external flow along the $y$ direction and the circulation due to a vortex somewhere near, or inside the disk. We assigned a radius $a$ to the disk and placed the origin at its center.

The determination of the critical velocity for vortex depinning can be made by looking for steady state solutions of the NLSE equation above. For $v_{\infty}$ below a certain critical value, dependent only on the disk radius, one is able to find stationary solutions representing a laminar flow. Above the critical velocity no stationary state exists, a state with a circulation around the disk can evolve to produce a vortex near the edge of the disk. The NLSE in $1 d$ was found to display the same type of behavior 15. 16], phase slips are dynamically produced behind an inhomogeneity if a large enough flow velocity is applied at infinity. We postpone the discussion of the numerical calculation to section IV.

\section{THE COLLECTIVE COORDINATE APPROACH}

Now we will follow Ref. [8] and study this problem using as a collective coordinate the $2 d$ location of the vortex in the flow region. We will ignore the role of the superfluid compressibility.

When we write $\psi=\sqrt{\rho} e^{i \phi}$, the NLSE yields a continuity equation and an Euler equation for the superfluid density $\rho$ and superfluid velocity $\mathbf{v}=\nabla \phi$. In the incompressible limit these equations become:

$$
\begin{gathered}
\nabla \cdot \mathbf{v}=0, \\
\frac{\partial \mathbf{v}}{\partial t}+(\mathbf{v} \cdot \nabla) \mathbf{v}=0 .
\end{gathered}
$$

If a set of vortices of charge $n_{i}= \pm 1$ is present at positions $\mathbf{R}_{i}$, we also have:

$$
\nabla \times \mathbf{v}=\sum_{i} 2 \pi n_{i} \delta\left(\mathbf{r}-\mathbf{R}_{i}\right)
$$

The boundary condition 3 becomes

$$
\mathbf{v} \cdot \hat{r}=0, \quad r=a .
$$

The problem of determining the velocity field from a given distribution of vortices and a fixed boundary condition at infinity is contained in equations 1 and 6 . They can be cast in the form of a $2 d$ electrostatic problem if we define the potential $\chi$ :

$$
\mathbf{v}=\nabla \chi \times \hat{z} .
$$

Eqs. 17 and 6 reduce to a Poisson's equation:

$$
\nabla^{2} \chi=-\sum_{i} 2 \pi n_{i} \delta\left(\mathbf{r}-\mathbf{R}_{i}\right)
$$

As for the boundary condition, Eq. 2 translates to $\chi \rightarrow \chi_{0}-v_{\infty} x$ at infinity and the disk becomes an equipotential of $\chi$ due to the impenetrability condition 0 . 
The vortex dynamics is contained in equation 5 . As is well known, inside an ideal and incompressible fluid the vortex follows the flow velocity at its center (excluding the singular contribution of the vortex itself to $\mathbf{v}$ ). This being simply Kelvin's theorem [17]:

$$
\frac{d \mathbf{R}}{d t}=\mathbf{v}(\mathbf{R}, t) .
$$

In the Appendix we show how to go from Eq. 司 to Eq. 10 .

We now derive the dynamics of a vortex of charge $n$ at position $\mathbf{R}$ outside the disk, with an applied flow at infinity, $\mathbf{v}_{\infty}=v_{\infty} \hat{y}$, by first writing the general solution of Eq. 9 as the sum of three terms:

$$
\begin{gathered}
\chi_{\mathrm{e}}(\mathbf{r})=-v_{\infty} x\left(1-a^{2} / r^{2}\right), \\
G_{0}(\mathbf{r}, \mathbf{R})=-n \ln |\mathbf{r}-\mathbf{R}|, \\
G_{I}(\mathbf{r}, \mathbf{R})=+n \ln \left|\mathbf{r}-\left(a^{2} / R^{2}\right) \mathbf{R}\right|-n \ln (r / a) .
\end{gathered}
$$
by:

The total flow, which obeys the topological constraint $\oint \mathbf{v} \cdot d \mathbf{l}=n$ for a path enclosing the disk at infinity, is given

$$
\mathbf{v}(\mathbf{r}, t)=\nabla_{\mathbf{r}}\left[\chi_{\mathrm{e}}(\mathbf{r})+G_{0}(\mathbf{r}, \mathbf{R})+G_{I}(\mathbf{r}, \mathbf{R})\right] \times \hat{z} .
$$

To find the flow velocity at the vortex position and the vortex equation of motion we use the symmetry properties of the Green's functions and obtain (excluding the singular contribution of $G_{0}$ ):

$$
\frac{d \mathbf{R}}{d t}=\nabla_{\mathbf{R}}\left[\chi_{\mathrm{e}}(\mathbf{R})+\frac{1}{2} G_{I}(\mathbf{R}, \mathbf{R})\right] \times \hat{z} .
$$

One can view this equation as Newton's equation for a massless object moving in a constant $B$-field along the $z$ direction plus an electrostatic potential set by $\chi_{\mathrm{e}}$ and $G_{I}$ (the applied flow plus the vortex images). Alternatively, the $X$ and $Y$ coordinates of the vortex can be thought of as conjugate variables with $\chi_{\mathrm{e}}+\frac{1}{2} G_{I}$ playing the role of the Hamiltonian.

The absence of a mass term is due to the incompressibility assumption, the effect of the compressibility is to introduce retardation effects in the response of the vortex to its external surroundings, which in turn gives rise to a frequency dependent mass term in the vortex equation of motion 18 . The issue of the vortex mass in a neutral superfluid was, until recently, the subject of many papers [19].

Fig. 1 shows the isolines of the Hamiltonian function, which correspond to the trajectories followed by a positive vortex in the incompressible limit. There one sees a saddle point to the right of the disk separating closed trajectories near the disk from open ones, of the same energy, further from it.

As it stands, Fig. 1 describes the dynamics of a vortex that is already outside the disk, but we want to describe the process of vortex depinning whereby the quantum of circulation, initially inside the disk, is pushed outwards by the flow in the form of a vortex. The problem we face is characteristic of the collective coordinate approach since we are using as variable the very object whose creation we want to describe. One way out of this dilemma is to recognize that the incompressibility hypothesis should hold only at a distance $\delta(\sim \xi)$ away from the obstacle. The nucleation process in fact happens, due to compressibility effects, within a coherence length from the disk, see Ref. [6] and Fig. 3 below.

If the parameter $v_{\infty}$ is such that the saddle point is inside this region dominated by the compressibility, one has the possibility of the nucleated vortex to appear in one of the open orbits to the right of the disk and be dragged along by the flow, as one observes in the simulation of the NLSE. Correspondingly, when the saddle point is outside the compressibility region any nucleated vortex would remain close to the disk, in one of the closed orbits. We would like to identify this situation with what happens when the velocity is below the critical velocity and the quantum of circulation remains trapped inside the disk.

We therefore find an estimate for the critical velocity from:

$$
\frac{\partial}{\partial X}\left[\chi_{\mathrm{e}}(\mathbf{R})+\frac{1}{2} G_{I}(\mathbf{R}, \mathbf{R})\right]_{(X, Y)=(a+\delta, 0)}=0
$$

and view $\delta$ as an adjustable parameter of order $\xi$. 
We obtain:

$$
v_{\mathrm{c}}=\frac{a^{2}(a+\delta)}{(a+\delta)^{4}-a^{4}}
$$

An alternative expression for the critical velocity is that used in Ref. [20]. One writes the NLSE as two equations for $\rho$ and $\phi$. One of the equations is a continuity equation, the other is:

$$
-\frac{\partial \phi}{\partial t}=-\frac{1}{2} \frac{\nabla^{2} \sqrt{\rho}}{\sqrt{\rho}}+\frac{1}{2}(\nabla \phi)^{2}+\rho-1 .
$$

In the steady state, $\partial \phi / \partial t$ must be a constant. The asymptotic region fixes this value and one finds:

$$
\frac{1}{2}\left(v_{\infty}^{2}-v^{2}\right)=-\frac{1}{2} \frac{\nabla^{2} \sqrt{\rho}}{\sqrt{\rho}}+\rho-1
$$

If one ignores the Laplacian in this equation one obtains a relation between $v$ and $\rho$. Above a certain value of $v$ one has $d[\rho(v) v] / d v<0$, this defines $v_{\max }=\sqrt{\left(v_{\infty}^{2}+2\right) / 3}$ as the maximum possible velocity inside the fluid. If at some point $v>v_{\max }$ a vortex will be nucleated so as to reduce the flow velocity at that point. We can estimate the highest velocity in the flow using the incompressible limit. If a quantum of circulation is present inside the disk, $v$ is maximum at the equator, and it is equal to $2 v_{\infty}+1 / a$. This gives the critical velocity as the maximum value of $v_{\infty}$ that ensures $v<v_{\max }$ everywhere in the flow:

$$
v_{\mathrm{c}}=\frac{3}{11}\left[-\frac{2}{a}+\sqrt{\frac{1}{3 a^{2}}+\frac{22}{9}}\right] .
$$

In the limit $a \rightarrow \infty$ one finds the critical velocity for vortex/antivortex pair nucleation, $\left(v_{\mathrm{c}}\right)_{\text {pair }}=\sqrt{2 / 11}$, quoted in Ref. [6].

In Fig. 2 we show the dependence of $v_{\mathrm{c}}$ on the disk radius from Eqs. 17 and 20. In the first case we used $\delta=0.5$.

\section{NUMERICAL SIMULATION}

To determine numerically the critical velocity for depinning we looked for the values of $v_{\infty}$ which allowed a steady state solution of Eq. 1. This solution, with the boundary condition of Eq. 2 at infinity, has $e^{-i v_{\infty}^{2} t / 2}$ as time dependence. The spatial dependence is then found as a solution of

$$
\frac{v_{\infty}^{2}}{2} \psi=-\frac{1}{2} \nabla^{2} \psi+\left(|\psi|^{2}-1\right) \psi
$$

with boundary conditions given by Eqs. 2 and 3 .

We wrote this equation using finite differences on a two dimensional grid and reduced the problem to a set of $N$ complex, nonlinear algebraic equations, for $N$ complex variables, the values of $\psi$ at the grid nodes. This was solved, starting from a trial state, using Newton's method [21]. The number of nodes varied depending on the disk radius so that we always had a minimum distance of $10 \xi$ between the disk boundary and the sides of the working area. The grid spacing used was $0.5 \xi$, but it was reduced to $0.25 \xi$ for disks of smaller radii to ensure a reasonable representation of the disk as a region of the square grid. The disk radii ranged from $0.5 \xi$ to $10 \xi$.

After a solution with a given value of $v_{\infty}$ was found, a new $v_{\infty}$, increased by 0.1 , was used in the boundary condition and in equation 21. The code was run again taking the available solution as trial state. When Newton's method failed to converge we took the last value of $v_{\infty}$ as a lower limit for the critical velocity.

The critical velocities so obtained are shown in Fig. 2. There one sees an increase of $v_{\mathrm{c}}$ with the disk radius, as both Eqs. 17 and 20 predict, and an apparent saturation at the value $0.5 c$, higher than the prediction of $\sqrt{2 / 11} c$ of Eq. 20. The value of $\delta$ that must be used in Eq. 17 to obtain this asymptotic value of $v_{\mathrm{c}}$ is $0.5 \xi$.

Above the critical velocity, if one uses as initial state a condensate with a quantum of circulation inside the disk, one can observe the creation of a vortex from the dynamics of the NLSE, Eq. 1. This equation was solved numerically using finite differences in a grid similar to the one described above. The condensate at time zero was evolved using a split-step algorithm 21] where the Laplacian part and the nonlinear part of the NLSE were used alternately. The time step was $0.1 \xi / c$. The condensate amplitude at four different times is shown in Fig. 3. There one observes the vortex formation in the region close to the disk equator and its subsequent movement downstream. After the vortex depinning, a vortex/antivortex pair is seen nucleating as in Ref. [6]. This happens because the value of $v_{\infty}=0.5 c$ used is also above the critical velocity for pair nucleation for that particular radius, $a=1 \xi$. 


\section{MACROSCOPIC QUANTUM TUNNELING}

Below the critical velocity an effective barrier prevents the circulation around the disk to become a vortex outside the disk. At zero temperature this barrier can only be overcome via Macroscopic Quantum Tunneling. In a thorough semiclassical treatment we would have to find the bounce in imaginary time that connects the condensate corresponding to the stationary, laminar flow, solution of the NLSE at $\tau=-\infty$ to a condensate containing a nucleated vortex near the disk at $\tau=0$, and back to the stationary state at $\tau=+\infty$. This bounce would be a nontrivial solution of the imaginary time NLSE [15]:

$$
\begin{aligned}
-\frac{\partial \psi}{\partial \tau} & =-\frac{1}{2} \nabla^{2} \psi+(\psi \bar{\psi}-1) \psi, & \psi(\mathbf{r},-\infty) & =\psi_{0}(\mathbf{r}) \\
\frac{\partial \bar{\psi}}{\partial \tau} & =-\frac{1}{2} \nabla^{2} \bar{\psi}+(\psi \bar{\psi}-1) \bar{\psi}, & \bar{\psi}(\mathbf{r},+\infty) & =\psi_{0}^{*}(\mathbf{r})
\end{aligned}
$$

Here, $\psi_{0}(\mathbf{r})$ is the stationary condensate that represents the laminar flow around the disk with the circulation trapped inside. The bounce solution would show, as imaginary time progresses, a vortex leaving the disk towards the flow and then returning back. The vortex position at $\tau=0$ would indicate the most favorable position inside the flow for the vortex to emerge.

Instead of retaining all degrees of freedom of the condensate in the calculation of the bounce one can use the collective coordinate approach of section III and focus on the dynamics of the vortex position. In this approach the bounce, or tunneling path, connects a closed orbit in the $X Y$ plane to its open counterpart having the same value of $E=\chi_{\mathrm{e}}+\frac{1}{2} G_{I}$. This is done by allowing the momentum $Y$ to become imaginary, see Fig. 4 . The logarithm of the WKB tunneling rate is then proportional to the $p \dot{q}$ part of the action that governs the vortex dynamics, Eq. 15. This action in real time is:

$$
S[\mathbf{R}]=h \rho_{0} \xi^{2} \int\left[Y \dot{X}-\chi_{\mathrm{e}}(\mathbf{R})-\frac{1}{2} G_{I}(\mathbf{R}, \mathbf{R})\right] d t
$$

The semiclassical tunneling rate is then:

$$
\Gamma_{\mathrm{WKB}} \propto \exp \left[-2 \pi \rho_{0} \xi^{2} \oint|Y(X, E)| d X\right],
$$

with $Y(X, E)$ being the trajectory in the $X Y$ plane that is a solution (with imaginary $Y$ ) of:

$$
\chi_{\mathrm{e}}(\mathbf{R})+\frac{1}{2} G_{I}(\mathbf{R}, \mathbf{R})=E .
$$

The argument in the exponential is $2 \pi$ times the number of bosons enclosed by the imaginary momentum trajectory. To compare, in the field theory for the condensate we find the tunneling rate from:

$$
\Gamma_{\mathrm{WKB}} \propto \exp \left[-\rho_{0} \xi^{2} \int \bar{\psi}_{\mathrm{b}} \frac{\partial \psi_{\mathrm{b}}}{\partial \tau} d \mathbf{r} d \tau\right],
$$

where $\psi_{\mathrm{b}}(\mathbf{r}, \tau)$ is the bounce solution of Eqs. 22 and 23 .

Whereas the semiclassical recipe for the tunneling of $\psi$ is well defined, in the collective coordinate treatment it is not clear between which pair of trajectories the tunneling will take place, or equivalently, which value of $E$ is to be used in Eq. 25. This ambiguity stems from the fact that the collective coordinate approach does not really describe the vortex nucleation process, instead it describes the tunneling of a preexisting vortex bound to the disk in a closed trajectory towards an open trajectory further inside the flow.

One can imagine doing the same kind of approximation used to obtain the formula for the critical velocity and simply admit that the tunneling will occur between the closed trajectory that reaches a distance $\delta$ from the disk and its open counterpart. This would correspond to the vortex being produced at the furthest possible distance inside the compressibility dominated region around the disk. This fixes the value of $E$ to be used in Eq. 25 and is consistent with the formula for the critical velocity, Eq. 17, since we then obtain $\Gamma_{\mathrm{WKB}} \rightarrow 1$ as $v_{\infty} \rightarrow v_{\mathrm{c}}$ (when the saddle point is exactly at a distance $\delta$ from the disk).

In Fig. 5 we show the dependence of the tunneling rate with the applied velocity, $v_{\infty}$, calculated using Eq. 25 for radii $a=1,2$ and $3 \xi$ and $\delta=0.5$ and $1.5 \xi$. One observes, for small values of $v_{\infty} / v_{c}$, that

$$
\Gamma \sim \exp \left[-C\left(v_{c} / v_{\infty}\right)^{\alpha}\right], \quad \alpha=2.8 \pm 0.1,
$$

is a good fit to all curves. 


\section{CONCLUSION}

We have analyzed the process of vortex depinning from a two dimensional obstacle due to an applied superflow. We discussed the process of dynamical depinning, above the critical velocity, and the process of depinning via MQT, at zero temperature and below the critical velocity, with a collective coordinate approach. The problems inherent to this approach were circumvented with the introduction of a length $\delta(\sim \xi)$ proportional to the size of the compressibility dominated region around the defect. A more thorough calculation of the critical velocity was made using the full NLSE and compared with the estimate based on the collective coordinate. The process of dynamical nucleation was demonstrated with a numerical simulation of the time dependent NLSE.

It is left for a future work to contrast the the semiclassical tunneling rate computed from Eq. 25 with the one obtained by solving the imaginary time NLSE.

Experiments on critical velocities of two dimensional neutral superflows past obstacles do not exist in the literature, nevertheless one can expect the calculations presented here to be applicable to layered superconductors in the superclean limit, when the pancake vortices are expected to follow the dynamics discussed in section III, see Ref. 22]. When

such systems are irradiated by heavy ions defects similar to the ones discussed here can be formed. Measurements of the critical current dependence with the temperature and applied magnetic field already exists, see for instance Ref. [23], but the dependence with the defect radius is still lacking, when obtained a comparison could be made with the results presented here.

\section{APPENDIX: KELVIN'S THEOREM}

Here we derive Kelvin's theorem, Eq. 10, from Eq. 5. We consider a flow field $\mathbf{v}(\mathbf{r}, t)$ and compute the rate of change of the circulation in a path $C(t)$ being dragged by the flow:

$$
\frac{d}{d t} \oint_{C(t)} \mathbf{v}(\mathbf{r}, t) \cdot d \mathbf{l}
$$

We must evaluate,

$$
\frac{1}{d t}\left[\oint_{C(t+d t)} \mathbf{v}(\mathbf{r}, t+d t) \cdot d \mathbf{l}-\oint_{C(t)} \mathbf{v}(\mathbf{r}, t) \cdot d \mathbf{l}\right] .
$$

The mapping between points in the $C(t)$ loop and points in the $C(t+d t)$ loop is

$$
\mathbf{r} \rightarrow \mathbf{r}+\mathbf{v}(\mathbf{r}, t) d t .
$$

Therefore an element $d \mathbf{l}$ in $C(t)$ is mapped to

$$
\begin{aligned}
\mathbf{r}+d \mathbf{l} & \rightarrow \mathbf{r}+d \mathbf{l}+\mathbf{v}(\mathbf{r}+d \mathbf{l}, t) d t \\
d \mathbf{l} & \rightarrow d \mathbf{l}+(d \mathbf{l} \cdot \nabla) \mathbf{v}(\mathbf{r}, t) d t
\end{aligned}
$$

The integral in the $C(t+d t)$ loop can then be written as

$$
\oint_{C(t)} \mathbf{v}[\mathbf{r}+\mathbf{v}(\mathbf{r}, t) d t, t+d t] \cdot\{d \mathbf{l}+(d \mathbf{l} \cdot \nabla) \mathbf{v}(\mathbf{r}, t) d t\},
$$

or, to lowest order in $d t$,

$$
\oint_{C(t)}\left\{\mathbf{v}+\partial_{t} \mathbf{v} d t+(d t \mathbf{v} \cdot \nabla) \mathbf{v}\right\} \cdot d \mathbf{l}+\oint_{C(t)} \mathbf{v} \cdot(d \mathbf{l} \cdot \nabla) \mathbf{v} d t .
$$

Finally we obtain for the derivative,

$$
\frac{d}{d t} \oint_{C(t)} \mathbf{v} \cdot d \mathbf{l}=\oint_{C(t)} \partial_{t} \mathbf{v} \cdot d \mathbf{l}+\oint_{C(t)}(\mathbf{v} \cdot \nabla) \mathbf{v} \cdot d \mathbf{l}+\oint_{C(t)} \frac{1}{2} \nabla v^{2} \cdot d \mathbf{l} .
$$

The first two integrals add up to zero by virtue of Eq. 5, whereas the third integral is identically zero as long as the path does not cross any singularity of $v^{2}$. 
The fact that the circulation is a constant along any path flowing with the fluid imply that the singular vortices must follow the flow velocity at their center. That is, Eq. 10 must hold.

[1] R.J. Donnelly, Quantized Vortices in Helium II (Cambridge Univ. Press, Cambridge, 1991).

[2] C.M. Muirhead, W.F. Vinen, F.R.S., and R.J. Donnelly, Phil. Trans. R. Soc. Lond. A 311, 433 (1984).

[3] P.C. Hendry, N.S. Lawson, P.V.E. McClintock, C.D.H. Williams and R.M. Bowley, Phys. Rev. Lett. 60, 604 (1988).

[4] J.C. Davis, J.Steinhauer, K. Schwab, Yu. M. Mukharsky, A, Amar, Y. Sasaki and R.E. Packard, Phys. Rev. Lett. 69, 323 (1992).

[5] G.G. Ihas, O. Avanel, R. Aarts, R. Salmelin and E. Varoquaux, Phys. Rev. Lett. 69, 327 (1992).

[6] T. Frisch, Y. Pomeau and S. Rica, Phys. Rev. Lett. 69, 1644 (1992).

[7] M. Stone and A.M. Srivastava, J. Low Temp. Phys. 102, 445 (1996).

[8] G.E. Volovik, JETP Lett. 15, 116 (1972).

[9] M.J. Stephen, Phys. Rev. Lett. 72, 1534 (1994).

[10] D.A. Gorokhov and G. Blatter, Phys. Rev. B 57, 3577 (1998).

[11] M.V. Feigel'man, V.B. Geshkenbein, A.I. Larkin and S. Levit, JETP Lett. 57, 711 (1993).

[12] E.B. Sonin and B. Horovitz, Phys. Rev. B 51, 6526 (1995).

[13] E.P. Gross, Nuovo Cimento 20, 454 (1961).

[14] L.P. Pitaevskii, Sov. Phys. JETP 13, 451 (1961).

[15] J.A. Freire, D.P. Arovas and H. Levine, Phys. Rev. Lett. 79, 5054 (1997).

[16] Vincent Hakim, Phys. Rev. E 55, 2835 (1997).

[17] G.K. Batchelor. An Introduction to Fluid Dynamics. Cambridge University Press, 1967.

[18] D.P. Arovas and J.A. Freire, Phys. Rev. B 55, 1068 (1997).

[19] Ji-Min Duan, Phys. Rev. B 48, 333 (1993); Q. Niu, P. Ao and D.J. Thouless, Phys. Rev. Lett. 72, 1706 (1994); E.B. Sonin et al., Phys. Rev. B 57, 575 (1998); G.E. Volovik, JETP Lett. 67, 528 (1998).

[20] C. Josserand, Y. Pomeau and S. Rica, Phys. Rev. Lett. 75, 3150 (1995).

[21] W.H. Press et al., Numerical Recipes (Cambidge Univ. Press, Cambridge, 1986).

[22] G. Blatter et al., Rev. Mod. Phys. 66, 1125 (1994).

[23] D. Prost et al., Phys. Rev. B 47, 3457 (1993).

FIG. 1. Isolines of $E=\chi_{\mathrm{e}}+\frac{1}{2} G_{I}$, see Eqs. 11 and 13. These lines also represent the trajectories of a positive vortex in the presence of an applied flow with $v_{\infty}=0.4 c$ along the $y$ direction. The disk radius is $a=1 \xi$. The solid lines are below the saddle point, the dashed lines are above.

FIG. 2. Critical velocity, in units of the speed of sound $c$, as a function of the disk radius $a$. The solid line was obtained from Eq. 17 with $\delta=0.5 \xi$ and the dashed line from Eq. 20. The squares are lower limits for $v_{c}$ obtained from the time independent NLSE, Eq. 21, with a grid spacing of $0.5 \xi$. The open circles used a grid spacing of $0.25 \xi$ (the two values coincide for $a=0.5 \xi$ ).

FIG. 3. Countour lines of the condensate amplitude obtained from a numerical integration of the time dependent NLSE, Eq. 1. The amplitude is zero inside the disk of radius $2.5 \xi$ and increases to the equilibrium value of $\sqrt{\rho_{0}}$ away from it. The initial state had a smooth circulation around the disk in addition to the applied flow in the $y$ (vertical) direction of $v_{\infty}=0.5 c$. The times are 14, 23, 29 and $34 \xi$ (c respectively for figures a), b), c) and d). In a) the vortex is beginning to form, in b) it is fully formed and being dragged by the flow, in c) one observes a vortex/antivortex pair (the antivortex to the left and the vortex to the right) beginning to form and fully formed in $\mathrm{d}$ ).

FIG. 4. Close up view of the saddle point of Fig. 1. Here the solid lines are the solutions of Eq. 26 with real $Y$ and the dashed lines are solutions with imaginary $Y$. These can also be viewed as the real and imaginary time trajectories of a positive vortex around the disk of radius $a=1 \xi$ when an applied flow of $v_{\infty}=0.4 c$ along the $y$ direction is present. The area enclosed by the dashed paths is proportional to the logarithm of the tunneling rate. 
FIG. 5. Logarithm of the semiclassical tunneling rate as a function of the applied flow velocity at infinity, calculated with Eq. 25. The value of $E$ used in that equation was chosen to describe a vortex tunneling from a distance $\delta$ from the disk towards an open trajectory, of same energy, further from it. $v_{c}$ was calculated using this value of $\delta$ in Eq. 17. The squares, circles and triangles correspond to disk radius $a=1,2$ and $3 \xi$ respectively. The solid symbols use $\delta=0.5 \xi$ and the open symbols use $\delta=1.5 \xi$. 


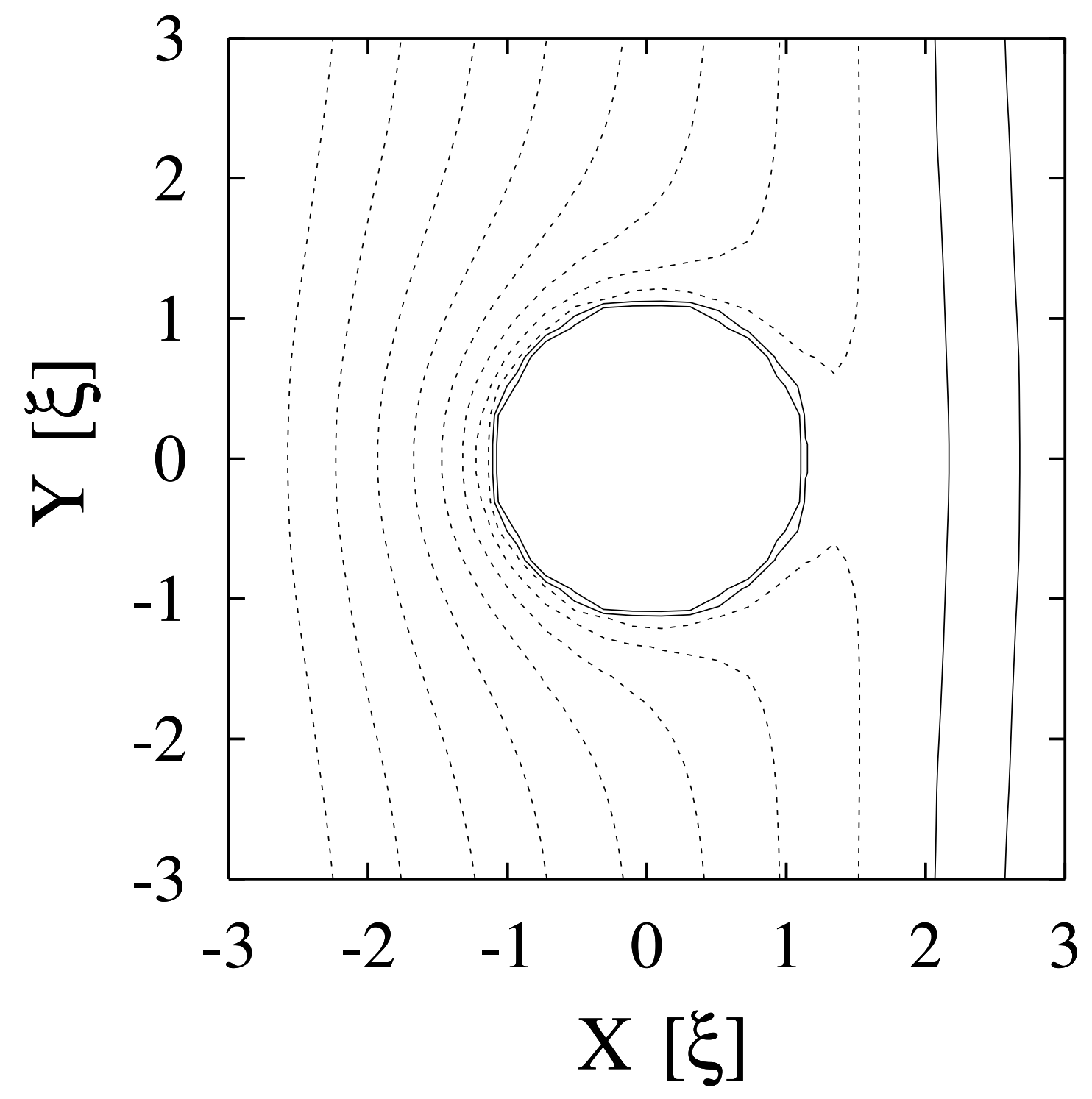




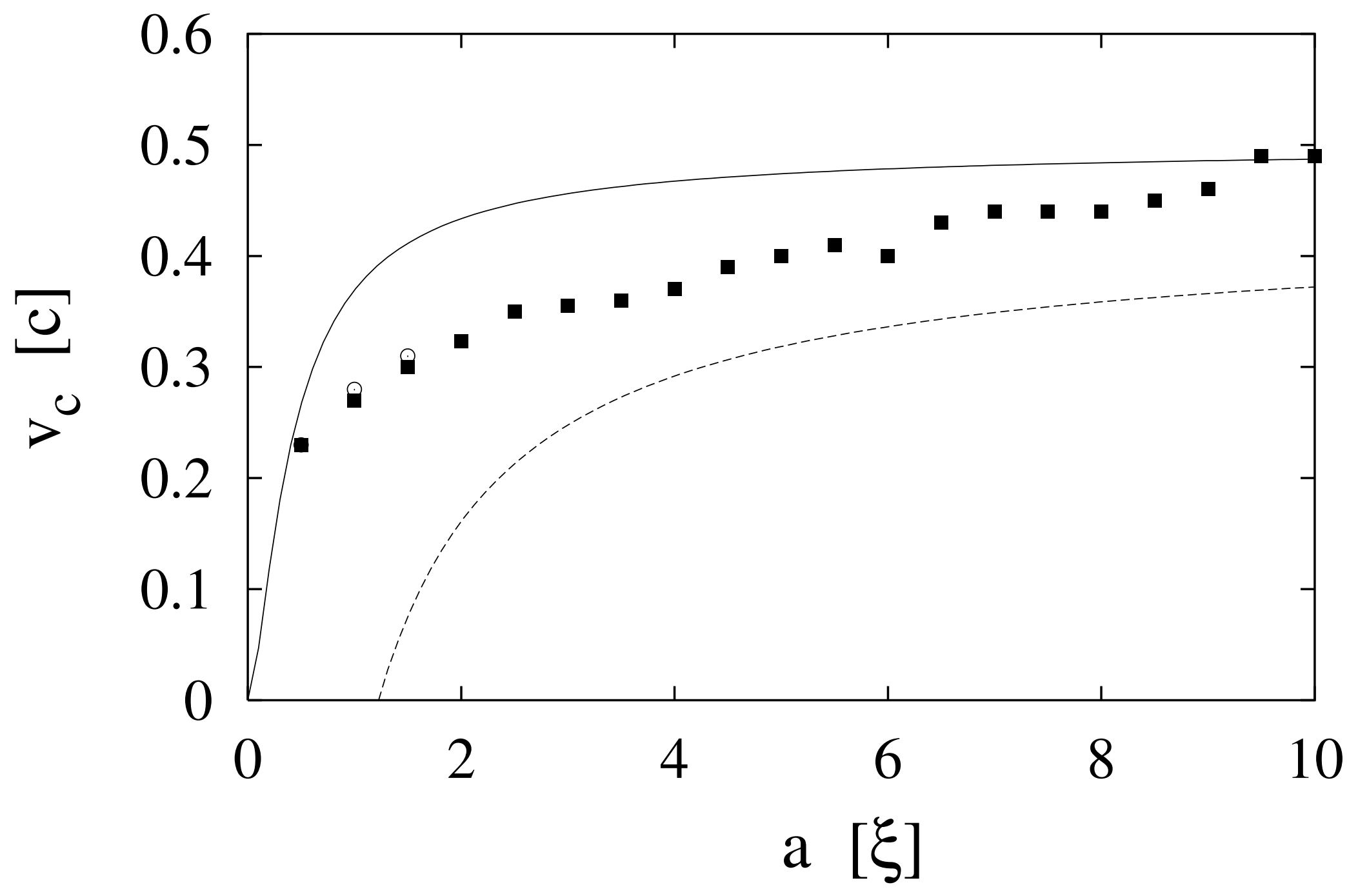



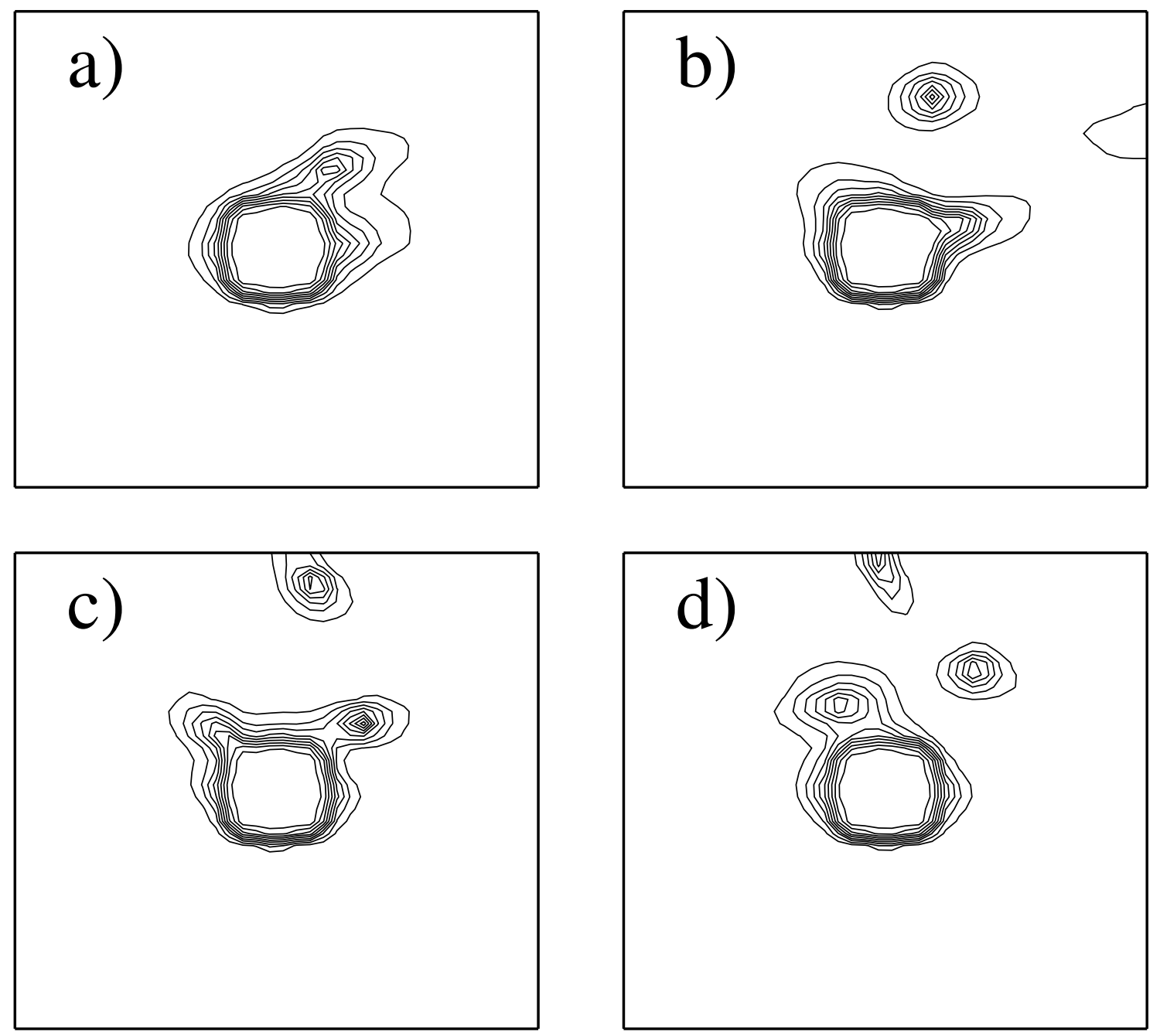


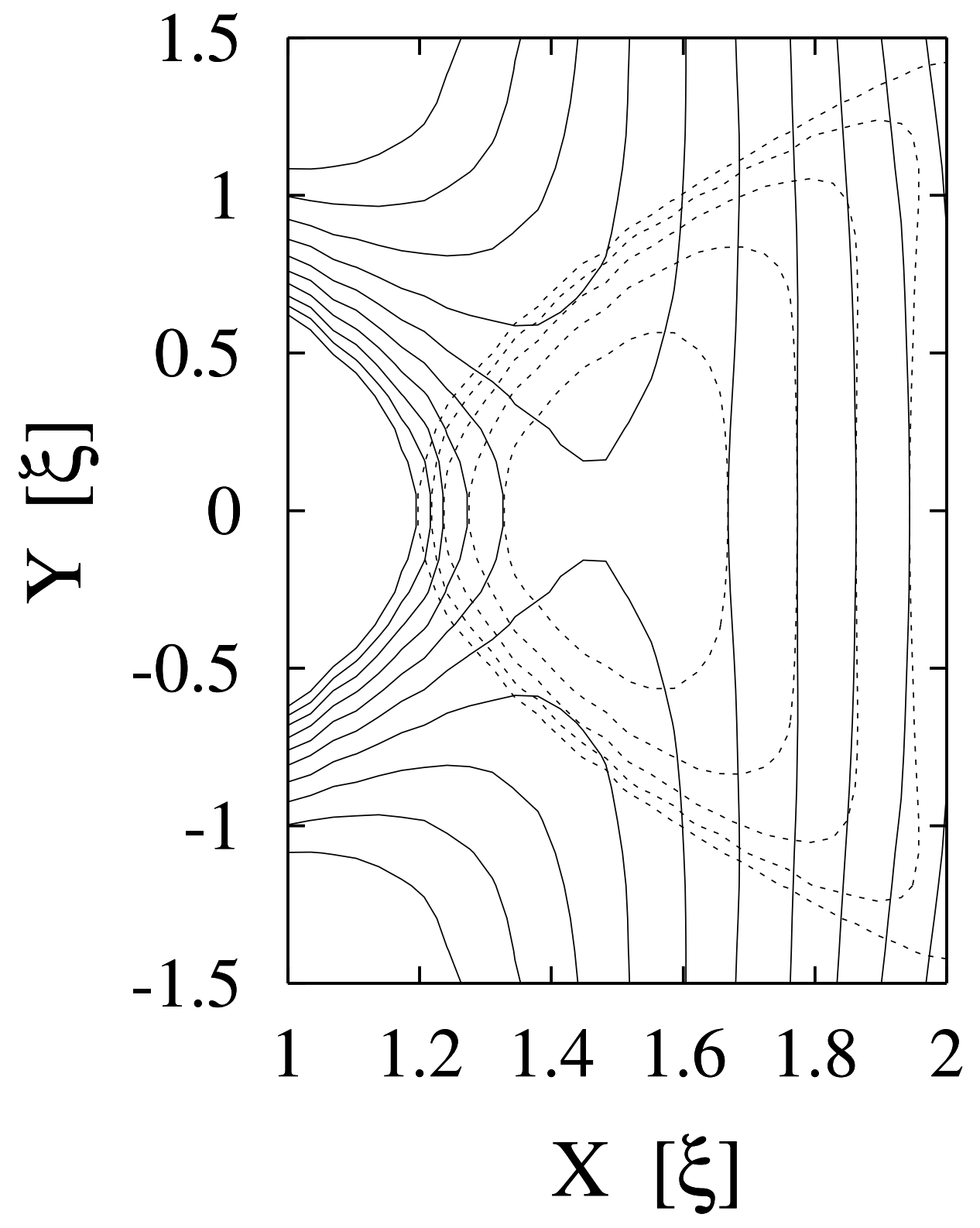




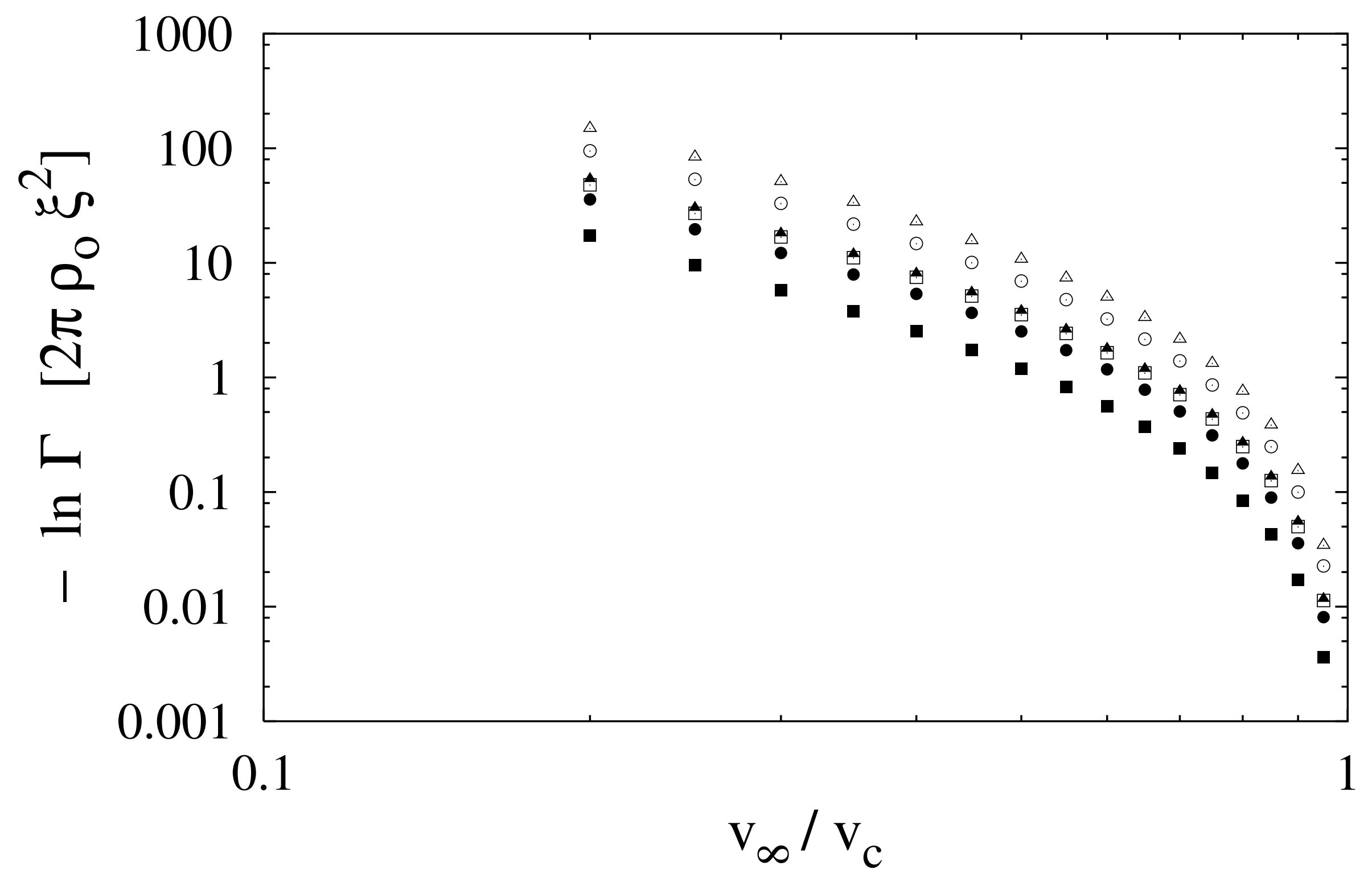

